\title{
Stereotype Threat Lowers Older Adults' Self-Reported Hearing Abilities
}

\author{
Sarah J. Barber Soohyoung Rain Lee \\ Department of Psychology, San Francisco State University, San Francisco, Calif., USA
}

\section{Key Words}

Stereotype threat · Hearing loss · Hearing impairment · Ageism · Old age

\begin{abstract}
Background: Although stereotype threat is a well-documented phenomenon, previous studies examining it in older adults have almost exclusively focused on objective cognitive outcomes. Considerably less attention has been paid to the impact of stereotype threat on older adults' subjective assessments of their own abilities or to the impact of stereotype threat in noncognitive domains. Objective: Older adults are stereotyped as having experienced not only cognitive declines, but physical declines as well. The current study tested the prediction that stereotype threat can negatively influence older adults' subjective hearing abilities. Methods: To test this, 115 adults (mean age 50.03 years, range 41-67) read either a positive or negative description about how aging affects hearing. All participants then answered a questionnaire in which they assessed their own hearing abilities. Results: The impact of stereotype threat on self-reported hearing was moderated by chronological age. Participants in their 40s and early 50s were unaffected by the stereotype threat manipulation. In contrast, participants in their late $50 \mathrm{~s}$ and 60 s rated their hearing as being subjectively worse when under stereotype threat. Conclusion: The current study provides a clear demonstration that stereotype threat
\end{abstract}

negatively impacts older adults' subjective assessments of their own abilities. It is also the first study to demonstrate an effect of stereotype threat within the domain of hearing. These results have important implications for researchers investigating age-related hearing decline. Stereotype threat can lead to overestimation of the prevalence of age-related hearing decline. It can also serve as a confounding variable when examining the psychosocial correlates of hearing loss. Because of this, researchers studying age-related hearing loss should aim to provide a stereotype threat-free testing environment and also include assessments of stereotype threat within their studies.

(c) 2015 S. Karger AG, Basel

\section{Introduction}

Hearing loss affects 48.1 million Americans, and its prevalence increases with age. For example, whereas $12.9 \%$ of people in their 40 s have some degree of hearing loss, the prevalence increases to $28.5 \%$ for people in their 50 s and to $44.9 \%$ for people in their 60 s. In fact, by the time people reach their 80 s, the prevalence of hearing loss has climbed to $89.1 \%$ [1]. This in turn has negative health implications; self-reported hearing loss is associated with impaired physical functioning, steeper rates of cognitive decline, and lower health-related quality of life [for a review, see ref. 2].

\section{KARGER}

E-Mail karger@karger.com

www.karger.com/ger
(C) 2015 S. Karger AG, Basel

0304-324X/15/0621-0081\$39.50/0
Sarah J. Barber

Department of Psychology

San Francisco State University, 1600 Holloway Avenue

San Francisco, CA 94132 (USA)

E-Mail barber@sfsu.edu 
Although hearing loss is primarily due to physiological changes, psychological factors may also play a role. For example, in the study by Levy et al. [3], older adults' stereotypes about aging were assessed by asking them to state the first five words or phrases that came to mind when thinking about 'old people'. Responses were then coded for their negativity, which could range from low (e.g., 'compassionate') to high (e.g., 'senile'). In addition, participants' hearing was screened, both during the initial assessment as well as 36 months later. Results revealed that older adults who provided more negative stereotypes about aging during the initial assessment did worse on the hearing assessment 36 months later (after controlling for initial hearing and other covariates). However, a limitation of this study is its correlational design. Although results are consistent with the notion that aging stereotypes adversely affect hearing, it is also possible that an unassessed factor (such as a family history of hearing loss) led to both negative expectations about aging as well as to declines in objective hearing over time.

To further examine the impact of aging stereotypes on hearing in this study, we examined whether experimentally manipulated levels of stereotype threat (rather than individual differences in stereotypic beliefs) affect older adults' hearing. Stereotype threat occurs when people find themselves in situations where their performance has the potential to confirm a negative self-relevant stereotype. In response to this, people often underperform on the task at hand and inadvertently confirm the stereotype they were trying to avoid [4]. For example, older adults are stereotyped as having poor memory abilities. When this stereotype becomes salient to them they tend to underperform on memory tests [for a review, see ref. 5].

Although stereotype threat has been shown to affect older adults' objective performance, its influence on older adults' subjective assessments of their own abilities is less clear. For example, Hess and Hinson [6] examined the impact of age-based stereotype threat on subjective memory abilities in adults who ranged in age from 24 to 86 years. According to stereotype threat theory, only people who self-identify with the threatened group should exhibit stereotype threat effects [e.g., see ref. 7]. Thus, the effects of age-based stereotype threat should be limited to older adults. However, results only partially supported this. Consistent with stereotype threat theory, Hess and Hinson [6] found that their manipulation increased concerns about memory independence, particularly amongst older adults (e.g., 'If I want to have a good memory I need to have others to help me remember'). However, in con- trast to stereotype threat theory, their manipulation had a strong negative effect on participants' beliefs about their own memory abilities regardless of chronological age (e.g., 'I can remember the things I need to'; 'I can find ways to improve my memory'). This age invariance suggests that the effect of their manipulation may not have been due to stereotype threat, but rather may have arisen from experimenter demand or self-stereotyping priming effects [for a review, see ref. 5]. ${ }^{1}$ Furthermore, although other research has also reported that stereotype threat lowers older adults' subjective perceptions of their own memory abilities $[8,9]$ and found that stereotype threat affects older adults' self-reported loneliness, extraversion, and health [10], to our knowledge, none of these studies have included younger or middle adult control groups. It is therefore possible that, as in Hess and Hinson [6], these effects arose for reasons other than stereotype threat.

The goal of the current study was to further examine the impact of stereotype threat on older adults' self-perceptions of their own abilities. We did this within the novel domain of hearing abilities. We hypothesized that stereotype threat about age-related hearing decline would lead older adults to self-report having poorer hearing abilities. Furthermore, to demonstrate that this predicted behavioral assimilation was due to stereotype threat, we included adults who ranged in age from 41 to 69 years. We expected the effects of our stereotype threat manipulation to be absent for middle-aged adults, but to emerge as people enter older adulthood.

\section{Patients and Methods}

\section{Participants}

Participants were recruited through Amazon's Mechanical Turk (mTurk), an online service that connects people willing to do tasks with people who need those tasks completed. Previous research has shown that data obtained from mTurk participants has high test-retest reliability and does not significantly differ from data obtained from in-person lab study participants $[11,12]$. The study was advertised as investigating how people evaluate their own hearing. Interested individuals indicated their age, birth year, educational attainment, and whether or not they had a diagnosed hearing problem (e.g., deafness, use of a hearing aid). Of the 960 individuals who expressed interest in the study, only the 144 who self-reported being between the ages of 41 and 69 years and having no diagnosed hearing problems were able to complete the study. Of these 144 participants, we eliminated 29 from the data set:

\footnotetext{
Hess and Hinson [6] also included objective measures of memory performance. Consistent with stereotype threat theory, the influence of changes in memory belief on performance was moderated by chronological age; effects were strongest for older adults in their 60 s.
}

Barber/Lee 
Table 1. Demographic characteristics as a function of stereotype threat condition

\begin{tabular}{lll}
\hline & $\begin{array}{l}\text { Positive no-threat } \\
\text { condition }\end{array}$ & $\begin{array}{l}\text { Negative stereotype } \\
\text { threat condition }\end{array}$ \\
\hline $\begin{array}{l}\text { Age, years } \\
\quad \text { Mean } \pm \text { SD }\end{array}$ & $\begin{array}{l}51.0 \pm 27.31 \\
\quad \text { Range }\end{array}$ & $\begin{array}{l}49.12 \pm 6.46 \\
41-65\end{array}$ \\
Sex & 21 & 28 \\
$\quad$ Men & 34 & 32 \\
$\quad$ Women & 51 & 55 \\
Race & 2 & 1 \\
$\quad$ Caucasian & 1 & 3 \\
$\quad$ Asian & & 1 \\
$\quad$ African American & & 10 \\
$\quad$ Other & 4 & 38 \\
Education, \% & 42 & 38 \\
$\quad$ High school diploma & 38 & 13 \\
$\quad$ Associate's degree/some college & 16 & 7.45 (of 9) correct \\
$\quad$ Bachelor's degree & 7.42 (of 9) correct & \\
$\quad$ Advanced degree & &
\end{tabular}

There were no significant differences between the two conditions for these demographic variables. (a) 16 with responses from repeat IP addresses, (b) 1 whose comments indicated having completed the study multiple times, (c) 5 who provided inconsistent demographic information (e.g., an incompatible birth year and age), (d) 3 whose comments indicated a hearing problem, (e) 1 who did not complete all questionnaires, (f) 1 nonfluent English speaker, (g) 1 who expressed suspicion about the threat manipulation, and (h) 1 outlier in the positive nothreat condition whose self-reported hearing was $>2.5 \mathrm{SD}$ below that of other participants in that condition. This left 115 participants. Of these, 60 were randomly assigned to the negative stereotype threat group and 55 to the positive no-threat group. Participants were on average 50.03 years old. See table 1 for more demographic information.

\section{Procedure}

Participants were randomly assigned to the negative stereotype threat group or to the positive no-threat group. The level of threat was manipulated by asking participants to read fictitious news articles (copies of which are available from the first author upon request). In the negative stereotype threat condition, the article stated that hearing loss begins in midlife, rapidly accelerates with age, and leads to a faster rate of cognitive decline. In the positive nothreat condition, the article stated that younger adults' heavy use of earbuds has led to an 'epidemic of teenage hearing loss' and because of this, the current generation of younger adults has more hearing problems than the typical older adult. Of note, we used a positive no-threat manipulation rather than a neutral no-threat manipulation in order to clearly separate the conditions in terms of the amount of threat the subjects experienced. After reading the article, participants in both conditions were told that the study was designed to test the article's conclusions and that they had been selected to participate because they were an older adult.

Stereotype Threat and Hearing
Participants next answered 26 questions about their own hearing abilities. These questions were adapted from the Hearing Handicap Inventory for the Elderly - Screening Version (e.g., 'Do you have difficulty hearing when someone speaks in a whisper?'; 'Does a hearing problem cause you difficulty when listening to TV or radio?' [13]) and from the Speech Spatial and Qualities of Hearing Scale (e.g., 'You are in conversation with one person in a room where there are many other people talking. Can you follow what the person you are talking to is saying?'; 'Can you tell how far away a bus or truck is from the sound?' [14]). Participants responded to each question using a sliding scale from 0 to 100 . The internal consistency of this scale was excellent; Cronbach's a was 0.96 (with 10 items reverse scored). To ease interpretation of responses, raw scores were transformed to standardized T-scores.

At the end of the study, participants completed a demographics questionnaire, and their vocabulary was assessed via a 9-question abbreviated version of the Nelson-Denny test [15]. Participants received USD 1.50 upon study completion.

\section{Results}

As can be seen in figure 1, the effect of our stereotype threat manipulation on participants' self-reported hearing abilities was moderated by chronological age. To demonstrate this, we conducted a moderation analysis using Hayes' [16] (model 1) PROCESS macro for SPSS. Within this analysis, we used 95\% bias-corrected confidence intervals based on 5,000 bootstrapped samples, 


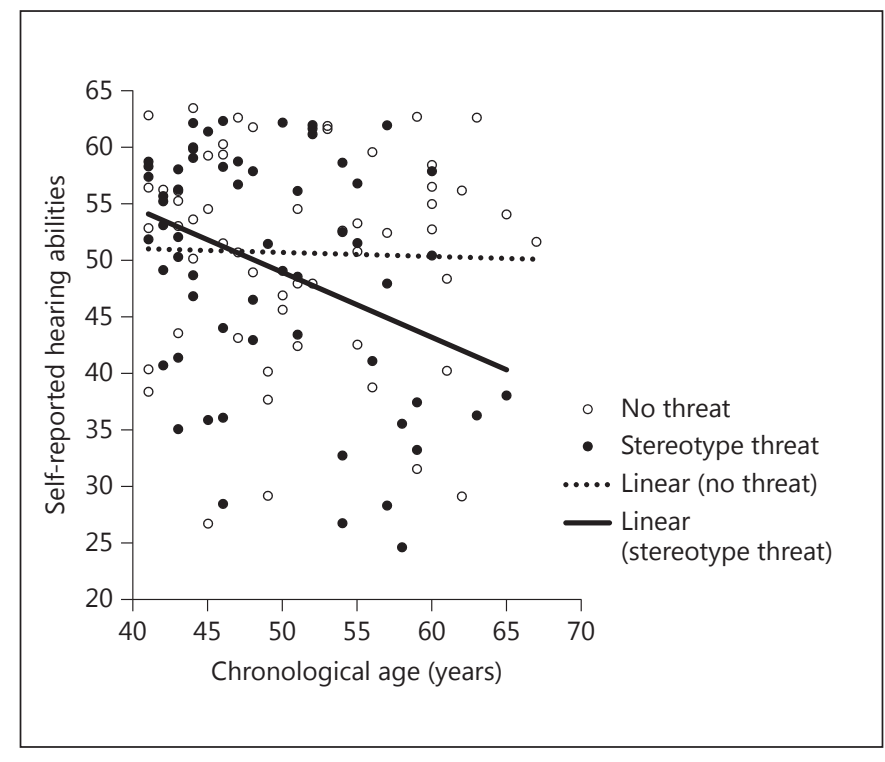

Fig. 1. The association between standardized self-reported hearing abilities and age as a function of stereotype threat condition. Raw self-reported hearing ability scores could theoretically range from 0 (poor hearing) to 2,600 (perfect hearing). To ease interpretation of results, raw scores were converted to standardized T-scores. This resulted in an average score of 50, with an SD of 10 . Results showed that there was no significant relationship between age and self-reported hearing abilities in the positive no-threat condition $(\mathrm{r}=-0.03, \mathrm{p}=0.84)$. In contrast, within the stereotype threat condition, there was a negative correlation between these variables: the older the participant, the lower their self-reported hearing abilities $(\mathrm{r}=-0.35, \mathrm{p}=0.006)$.

and mean centered the moderator and interaction term. Overall, this model was significant $[\mathrm{F}(3,111)=3.00$, $\mathrm{p}=$ $\left.0.03, \mathrm{R}^{2}=0.08\right]$. Although there was no significant direct effect of our stereotype threat manipulation on self-reported hearing $\left[t(111)=-0.96, p=0.34, \eta^{2}=0.008\right]$, there was a significant interaction between our stereotype threat manipulation and age in predicting self-reported hearing $\left[\mathrm{t}(111)=-2.02, \mathrm{p}=0.046, \eta^{2}=0.035\right]$ (which is indicative of moderation). Follow-up simple slopes analyses showed that stereotype threat did not affect self-reported hearing for participants who were 1 SD below the mean age of our sample [i.e., 43.10 years old; $t(111)=$ $\left.0.75, p=0.45, \eta^{2}=0.005\right]$, or who were at the mean age of our sample [i.e., 50.03 years old; $\mathrm{t}(111)=-0.96, \mathrm{p}=$ $\left.0.34, \eta^{2}=0.008\right]$. In contrast, stereotype threat was associated with significantly lower self-reported hearing for participants whose age was $1 \mathrm{SD}$ above the mean of our sample [i.e., 56.94 years old; $\mathrm{t}(111)=-2.10, \mathrm{p}=0.038$, $\left.\eta^{2}=0.038\right]$.

\section{Discussion}

The prevalence of hearing loss increases with age [1]. Because of this, some people incorrectly assume that all older adults have poor hearing abilities. For example, in one survey, 33\% of older Americans stated that they had interacted with someone who assumed that they could not hear well simply because of their age [17]. In this study, we tested the impact of this stereotype on subjective hearing. Results revealed that stereotype threat about age-related hearing declines negatively impacted self-reported hearing, but only for participants in their late $50 \mathrm{~s}$ or 60s. In contrast, stereotype threat about age-related hearing decline did not affect the middle-aged individuals in our sample who were in their 40 s or early 50 s.

The age specificity of our results suggests that they arose due to stereotype threat. According to stereotype threat theory, effects of threat should be limited to individuals who self-identify with the threatened group [8]. Thus, age-based stereotype threat should only affect people who self-identify as an older adult, which is more likely when people reach their late 50s and 60s. Our results are consistent with this and also rule out alternate explanations, such as experimenter demand or self-stereotyping [for a review, see ref. 5], which would have been associated with age-invariant outcomes. Furthermore, our results are the first to clearly show that older adults' selfassessments of their own abilities can be negatively affected by stereotype threat.

Although the current study is also the first to demonstrate an effect of stereotype threat within the domain of hearing, one limitation is that we did not include objective hearing measures. It is therefore not clear whether stereotype threat similarly lowers objective hearing performance, or whether the magnitude of stereotype threat effects varies between subjective and objective hearing performance outcomes. Of note, previous research has shown that for people younger than 70 years of age, the prevalence of subjective hearing problems is higher than the prevalence of objective hearing problems [18]. Furthermore, research has also shown that self-reports of hearing abilities can be affected by nonphysiological factors, such as cognitive abilities, educational attainment, and personality $[18,19]$. Taken together, these findings suggest that subjective hearing complaints may be more susceptible to psychological variables such as stereotype threat than objective hearing problems. However, future research is needed to examine this issue.

The current findings have implications for researchers studying age-related hearing loss. First, when older adult 
participants are aware that a study is investigating age differences in hearing abilities, they may experience stereotype threat. This in turn will lower their self-reported hearing and artificially increase the ability gap that is observed between the younger and older adult participants. It could also lead to overestimation of the prevalence of age-related hearing decline. To ameliorate these problems, we recommend that researchers avoid discussing age, or the association of age with hearing loss, prior to administering hearing assessments.

Second, researchers should keep in mind that stereotype threat can affect a wide variety of outcomes and may serve as a confounding variable when examining the psychosocial correlates of hearing loss. For example, there is a positive correlation between self-reported hearing loss and loneliness, which is presumed to be due to hearing loss-associated communication problems [e.g., see ref. 20]. However, within this relationship, stereotype threat is a potential confounder; the current results show that stereotype threat increases the likelihood of older adults reporting hearing problems, and a study by Coudin and Alexopoulos [10] suggests that stereotype threat also increases older adults' feelings of loneliness. Thus, when examining the psychosocial correlates of hearing loss, it is essential that researchers ensure that stereotype threat is minimized and/or measured.

\section{Acknowledgment}

This work was supported by a grant from the National Institute on Aging (grant number R01-AG046464) to S. Barber.

\section{References}

1 Lin FR, Niparko JK, Ferrucci L: Hearing loss prevalence in the United States. Arch Intern Med 2011;171:1851-1853.

2 Bainbridge KE, Wallhagen MI: Hearing loss in an aging American population: extent, impact, and management. Annu Rev Public Health 2014;35:1-39.

3 Levy BR, Slade MD, Gill TM: Hearing decline predicted by elders' stereotypes. J Gerontol B Psychol Sci Soc Sci 2006;61:82-87.

4 Steele CM: A threat in the air: how stereotypes shape intellectual identity and performance. Am Psychol 1997;52:613-629.

5 Barber SJ, Mather M: Stereotype threat in older adults: when and why does it occur, and who is most affected?; in Verhaeghen P, Hertzog C (eds): The Oxford Handbook of Emotion, Social Cognition, and Problem Solving during Adulthood. Oxford, Oxford University Press, 2014, pp 302-320.

6 Hess TM, Hinson JT: Age-related variation in the influences of aging stereotypes on memory in adulthood. Psychol Aging 2006;21:621625.

7 Schmader T: Gender identification moderates stereotype threat effects on women's math performance. J Exp Soc Psychol 2002; 38:194-201.
8 Hess TM, Hinson JT, Hodges EA: Moderators and mechanisms underlying stereotype threat effects on older adults' memory performance. Exp Aging Res 2009;35:153-177.

9 Bouazzaoui B, Follenfant A, Ric F, Fay S, Croizet J, Atzeni T, Taconnat L: Ageing-related stereotypes in memory: when the beliefs come true. Memory 2015, Epub ahead of print.

10 Coudin G, Alexopoulos T: 'Help me! I'm old!' How negative aging stereotypes create dependency among older adults. Aging Ment Health 2010;14:516-523.

11 Buhrmester M, Kwang T, Gosling SD: Amazon's Mechanical Turk: a new source of inexpensive, yet high-quality, data? Perspect Psychol Sci 2011;6:3-5.

12 Paolacci G, Chandler J, Ipeirotis PG: Running experiments on Amazon Mechanical Turk. Judgm Decis Making 2010;5:411-419.

13 Ventry IM, Weinstein BE: Identification of elderly people with hearing problems. ASHA 1983;25:37-47.

14 Gatehouse S, Noble W: The Speech, Spatial and Qualities of Hearing Scale (SSQ). Int J Audiol 2004:43:85-99.
15 Brown JI, Fishco VV, Hanna GS: NelsonDenny Reading Test, Forms G and H. Itasca, Riverside, 1993.

16 Hayes AF: PROCESS: a versatile computational tool for observed variable mediation, moderation, and conditional process modeling. Manuscript submitted for publication, 2012.

17 Palmore E: The ageism survey first findings. Gerontologist 2001;41:572-575.

18 Kiely KM, Gopinath B, Mitchell P, Browning CJ, Anstey KJ: Evaluating a dichotomized measure of self-reported hearing loss against gold standard audiometry: prevalence estimates and age bias in a pooled national data set. J Aging Health 2012;24:439-458.

19 Cox RM, Alexander GC, Gray GA: Personality, hearing problems, and amplification characteristics: contributions to self-report hearing aid outcomes. Ear Hear 2007;28:141162.

20 Kramer SE, Kapteyn TS, Kuik D, Deeg DJH: The association of hearing impairment and chronic diseases with psychosocial health status in older age. J Aging Health 2002;14:122137. 